\title{
Chaos in coupled Kerr-nonlinear parametric oscillators
}

\author{
Hayato Goto $\odot^{*}$ and Taro Kanao $\odot$ \\ Frontier Research Laboratory, Corporate Research \& Development Center, Toshiba Corporation, 1, \\ Komukai Toshiba-cho, Saiwai-ku, Kawasaki-shi, 212-8582, Japan
}

(Received 7 October 2021; accepted 9 December 2021; published 20 December 2021)

\begin{abstract}
A Kerr-nonlinear parametric oscillator (KPO) can generate a quantum superposition of two oscillating states, known as a Schrödinger cat state, via quantum adiabatic evolution and can be used as a qubit for gate-based quantum computing and quantum annealing. In this work, we investigate complex dynamics, i.e., chaos, in two coupled nondissipative KPOs at a few-photon level. After showing that a classical model for this system is nonintegrable and consequently exhibits chaotic behavior, we provide quantum counterparts for the classical results, which are quantum versions of the Poincaré surface of section and its lower-dimensional version defined with time integrals of the Wigner and Husimi functions and also the initial and long-term behavior of out-of-timeordered correlators. We conclude that some of them can be regarded as quantum signatures of chaos, together with energy-level spacing statistics (conventional signature). Thus, the system of coupled KPOs is expected to offer not only an alternative approach to quantum computing but also a promising platform for the study on quantum chaos.
\end{abstract}

DOI: 10.1103/PhysRevResearch.3.043196

\section{INTRODUCTION}

Computational basis states for quantum computing are usually taken from quantized energy levels. An alternative approach is to use oscillator states consisting of multiple quanta (photons or phonons). In this approach, multiple number states are used to represent each basis state, like logical qubits for quantum error correction [1]. Thus the oscillator approach to quantum computing will offer qubits robust to noise or hardware-efficient quantum error-correcting codes, which are known as bosonic codes [2-9].

One of such oscillator approaches is to use parametric oscillators. Their two stable oscillating states can be used for qubits. There are two types of parametric-oscillator approach: Dissipative and Kerr. In the dissipative-type approach, parametrically two-photon driven oscillators with large twophoton loss (larger than single-photon loss) are used for qubits $[10,11]$. Such a two-photon dissipative parametric oscillator becomes a Schrödinger cat state (superposition of two oscillating states) as a steady state [10-12], which has been demonstrated experimentally using superconducting circuits [13-15]. Since this type of qubit is insensitive to bit-flip errors [15], this approach is expected to be useful for fault-tolerant quantum computation $[16,17]$.

In the Kerr-type approach, parametrically two-photon driven oscillators with large Kerr nonlinearity $[18,19]$, which

\footnotetext{
*hayato1.goto@toshiba.co.jp

Published by the American Physical Society under the terms of the Creative Commons Attribution 4.0 International license. Further distribution of this work must maintain attribution to the author(s) and the published article's title, journal citation, and DOI.
}

we call Kerr-nonlinear parametric oscillators (KPOs) [20,21], are used for qubits. Low-loss KPOs have recently been realized experimentally using superconducting circuits [22-24]. An ideal KPO is lossless (nondissipative), and it can generate a Schrödinger cat state from the vacuum state via quantum adiabatic evolution (quantum bifurcation) $[3,20]$. Moreover, a network of KPOs can solve a combinatorial optimization problem (ground-state search in the Ising model) by adiabatic quantum computation [25-27] or quantum annealing $[28,29]$, the final state of which is a highly entangled state, a superposition of many-mode coherent states corresponding to two optimal solutions [20]. Quantum annealing using KPOs has been developed in the past 5 years [30-35]. The KPOs can also be used for qubits in gate-based quantum computing [21,36-40]. Since the KPO qubit, also known as a Kerr-cat qubit, is robust against bit-flip errors, like the above dissipative-type qubit, fault-tolerant quantum computation using KPOs has been developed [41,42]. The KPO has also offered physically interesting topics, such as nonclassical traveling-state generation $[43,44]$, quantum heating leading to Boltzmann sampling [45], steady-state entanglement generation [46,47], and phase transition [48,49].

In this work, we investigate nonlinear dynamics of coupled KPOs from the viewpoint of chaos [50,51]. The KPO, which is nondissipative in an ideal case, is more desirable for the study on chaos than the two-photon dissipative parametric oscillator (and also optical parametric oscillators [21,52], another dissipative type), because dissipation inevitably introduces noise (so-called quantum noise) [12,53,54], and such stochastic noise is undesirable for the study on chaos [50]. Nonlinear dynamics of a KPO have been studied [18,55]. To our knowledge, however, the coupled-KPO case has not been explored so far, though it has been suggested that the studies on chaos in a KPO network would be interesting [20]. 
(Chaos in a simplified classical model for the KPO network has also been suggested in the proposal of a quantum-inspired algorithm called simulated bifurcation [56], but it has not been investigated in detail.) Here we treat a system of two nondissipative KPOs with time-independent parameters as the simplest example sufficient for the study on chaos. (Similar studies on more KPOs are an interesting next step.)

This paper is organized as follows. In Sec. II, we introduce the quantum and classical models for the system. In Sec. III, we show our results for the classical model, where the nonintegrability of this model is shown by the Poincaré surface of section (SOS) [50,51] and its lower-dimensional version, and the sensitivity to initial conditions is also shown. These indicate chaos in the classical model. In Sec. IV, we provide our results for the quantum model, where the SOS and its lower-dimensional version are extended to quantum cases using the Wigner and Husimi functions [12,51,57], and the initial-condition sensitivity is also examined using outof-time-ordered correlators (OTOCs) [58-69]. Energy-level spacing statistics, which are a conventional quantum signature of chaos [51,70-73], are also discussed. Finally, we summarize our results in Sec. V.

\section{MODELS FOR TWO COUPLED KPOS}

The quantum and classical models for the KPO network have been introduced in Ref. [20] and well summarized in Ref. [21]. In the following, we provide these models in the case of two KPOs.

\section{A. Quantum model}

The quantum model for two KPOs is given by the following Hamiltonian:

$$
\begin{gathered}
H=H_{1}+H_{2}+H_{\mathrm{I}}, \\
H_{i}=\hbar \frac{K}{2} a_{i}^{\dagger 2} a_{i}^{2}-\hbar \frac{p_{i}}{2}\left(a_{i}^{2}+a_{i}^{\dagger 2}\right)+\hbar \Delta a_{i}^{\dagger} a_{i}, \\
H_{\mathrm{I}}=-\hbar \xi_{0}\left(a_{1}^{\dagger} a_{2}+a_{2}^{\dagger} a_{1}\right),
\end{gathered}
$$

where $a_{i}$ and $p_{i}$ are the annihilation operator and the parametric pump amplitude, respectively, for the $i$ th KPO; $K$ is the Kerr coefficient; $\Delta$ is the detuning of the KPO resonance frequency from half the pump frequency; $\xi_{0}$ is the coupling strength between the two KPOs; and $\hbar$ is the reduced Planck constant. In this work, we assume that $K, p_{i}, \Delta$, and $\xi_{0}$ are nonnegative.

\section{B. Classical model}

The corresponding classical model is derived by replacing the annihilation operator $a_{i}$ with a dimensionless complex amplitude $\alpha_{i}=x_{i}+i y_{i}$ (classical approximation) in the Heisenberg equations of motion for $a_{i}[20,21]$. Thus we obtain the equations of motion in the classical model:

$$
\begin{gathered}
\frac{d x_{i}}{d t}=\frac{\partial H_{\mathrm{c}}}{\partial y_{i}}=\left[K\left(x_{i}^{2}+y_{i}^{2}\right)+p_{i}+\Delta\right] y_{i}-\xi_{0} y_{j}, \\
\frac{d y_{i}}{d t}=-\frac{\partial H_{\mathrm{c}}}{\partial x_{i}}=-\left[K\left(x_{i}^{2}+y_{i}^{2}\right)-p_{i}+\Delta\right] x_{i}+\xi_{0} x_{j},
\end{gathered}
$$

$$
\begin{aligned}
H_{\mathrm{c}}(\mathbf{x}, \mathbf{y})= & \sum_{i=1,2}\left[\frac{K}{4}\left(x_{i}^{2}+y_{i}^{2}\right)^{2}-\frac{p_{i}}{2}\left(x_{i}^{2}-y_{i}^{2}\right)\right. \\
& \left.+\frac{\Delta}{2}\left(x_{i}^{2}+y_{i}^{2}\right)\right]-\xi_{0}\left(x_{1} x_{2}+y_{1} y_{2}\right),
\end{aligned}
$$

where $j \neq i$. Note that the equations of motion are no longer Newtonian mechanical ones, but still canonical Hamiltonian equations of motion [74]. In the following, we use "energy" for the value of the Hamiltonian.

For convenience, we introduce the potential energy $V_{\mathrm{c}}(\mathbf{x})$ defined by the minimum of $H_{\mathrm{c}}(\mathbf{x}, \mathbf{y})$ with respect to $\mathbf{y}$. Here we assume $\xi_{0}^{2}<p_{1} p_{2}$, which is the stability condition for $\mathbf{y}$ at the origin. Then, the Hamiltonian has a single minimum with respect to $\mathbf{y}$ at $\mathbf{y}=\mathbf{0}$. Thus the potential is given by $H_{\mathrm{c}}(\mathbf{x}, \mathbf{0})$, that is,

$$
V_{\mathrm{c}}(\mathbf{x})=\sum_{i=1,2}\left(\frac{K}{4} x_{i}^{4}-\frac{p_{i}-\Delta}{2} x_{i}^{2}\right)-\xi_{0} x_{1} x_{2} .
$$

This implies that the coupled-KPO model may resemble quartic oscillator models [75-78]. But here we focus on KPOs and leave the interesting relation between KPOs and quartic oscillators for future work.

\section{Parameter setting}

In this work, we take the following values for the above parameters:

$$
\begin{gathered}
\hbar=K=1, \\
p_{1}=3 K, \\
p_{2}=\pi K, \\
\Delta=0, \\
\xi_{0}=0,0.3 K, \text { or } K .
\end{gathered}
$$

Here Eq. (8) means that the units of energy and frequency are defined as $\hbar K$ and $K$, respectively (thus the unit of time is $K^{-1}$ ). The pump amplitudes around $3 K$ lead to mean photon numbers around 3 , because the mean photon numbers for oscillating states are given by $p_{i} / K$ when $\Delta=0$ [20]. We choose such small values because we are interested in the dynamics at a few-photon level. Also, we set $p_{2}$ to $\pi K$ such that $p_{2} \simeq p_{1}$ but $p_{2} / p_{1}$ becomes an irrational number, because then the ratio between the periods of the two KPOs in the decoupled case $\left(\xi_{0}=0\right)$ is irrational, and the dynamics become relatively complex. The three values of $\xi_{0}$ correspond to regular (integrable), intermediate, and chaotic (nonintegrable) cases, respectively, as shown in the next section. (Note that when $\xi_{0}=0$, that is, the two KPOs are decoupled, then the Hamiltonian for each KPO is conserved, and the system is integrable by definition [79].)

The potential energy $V_{\mathrm{c}}(\mathbf{x})$ with the above parameters is shown in Fig. 1. There is a minimum in each quadrant and a maximum at the origin. Thus the origin is unstable. In this work, we investigate the dynamics started around the origin (around the vacuum state in the quantum case). 
(a)
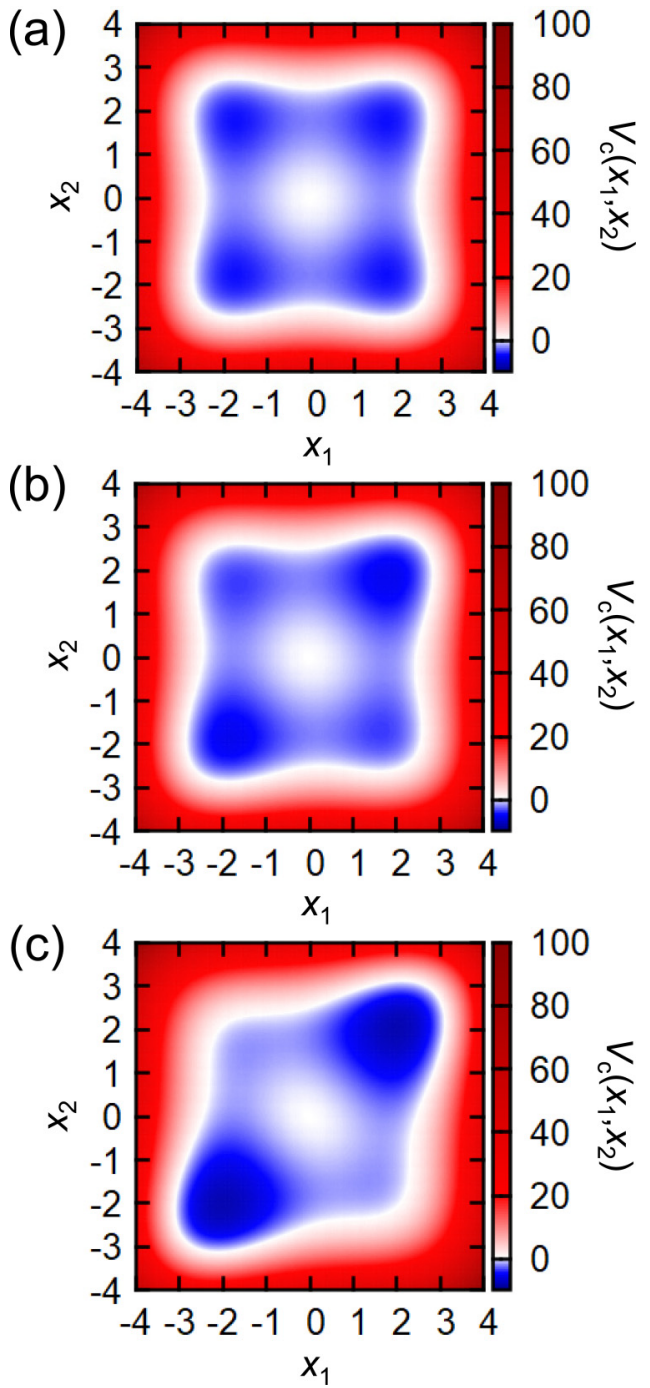

FIG. 1. Potential energy $V_{\mathrm{c}}(\mathbf{x})$ in $\mathrm{Eq}$ (7). (a) $\xi_{0}=0$. (b) $\xi_{0}=0.3 K$. (c) $\xi_{0}=K$. The other parameters are set as Eqs. (8)-(11).

\section{CHAOS IN THE CLASSICAL MODEL}

\section{A. SOS}

We start with nonintegrability of the classical model. To check nonintegrability, the Poincaré SOS [50,51] is useful, in particular for systems with two degrees of freedom (fourdimensional phase space), like the present system. The SOS in the phase space is defined by the section of the energy surface $H_{\mathrm{c}}(\mathbf{x}, \mathbf{y})=E$ ( $E$ is a constant $)$ by a plane, e.g., $y_{2}=0$. In the case of two degrees of freedom (four-dimensional phase space), the object consisting of the intersection points between the SOS and a trajectory, which we call the SOS plot, is twodimensional in general. If the system is integrable, however, there is another constant of motion [79], and consequently the SOS plot must be one-dimensional. Thus we can check whether the system is integrable or not by the dimension of the SOS plot.

Figure 2 shows the SOS plots in the classical model. The SOS plot is one-dimensional [80] (integrable and regular) in the decoupled case $\left(\xi_{0}=0\right)$ [Fig. 2(a)], two-dimensional (nonintegrable and chaotic) in the strong-coupling case

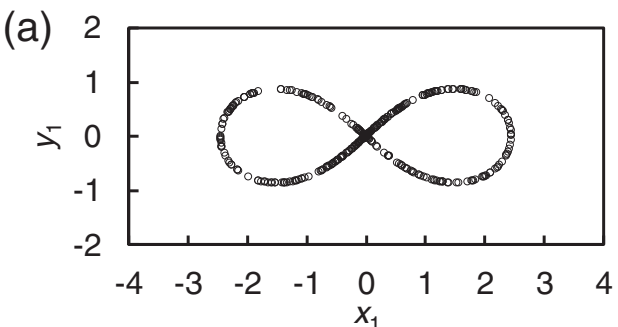

(b)
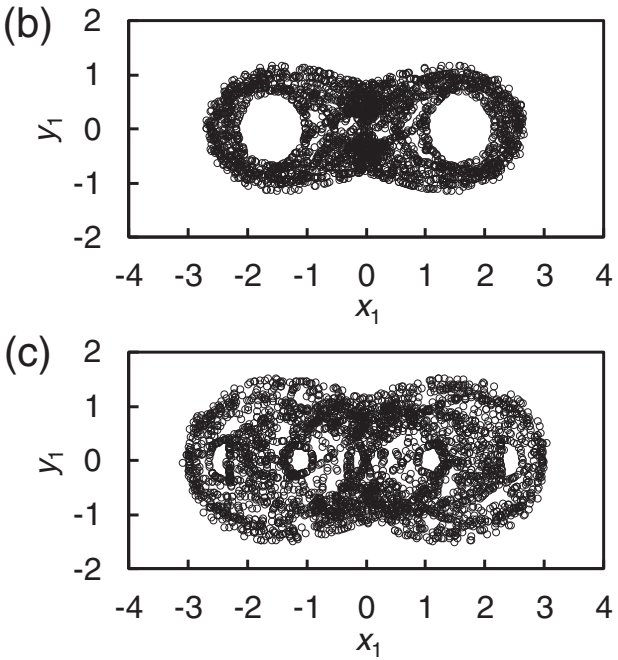

FIG. 2. SOS plots in the classical model given by Eqs. (4)-(6). Circles are obtained by plotting $\left(x_{1}, y_{1}\right)$ when trajectories in numerical simulation cross the plane $y_{2}=0$. (a) $\xi_{0}=0$. (b) $\xi_{0}=0.3 \mathrm{~K}$. (c) $\xi_{0}=K$. The other parameters are set as Eqs. (8)-(11). See Appendix A for details.

$\left(\xi_{0}=K\right)$ [Fig. 2(c)], and intermediate in the intermediate case $\left(\xi_{0}=0.3 K\right)$ [Fig. 2(b)], as expected.

\section{B. Momentum plot at a minimum of potential (MPMP)}

To demonstrate the nonintegrability more clearly, here we introduce another plot, which we call the MPMP. Instead of the plane $y_{2}=0$ for the SOS, here we fix the two positions, $x_{1}$ and $x_{2}$, at a minimum of the potential $V_{\mathrm{c}}(\mathbf{x})$ and plot the momenta in the $y_{1} y_{2}$ plane. We focus on a potential minimum, because at such a point, the energetically allowable region of the momenta becomes the largest, and that will be desirable for visualization. (For such a plot, any point can be chosen as long as the probability that the trajectory comes near it is nonzero. If the point is not a potential minimum, however, the energetically allowable region becomes smaller, and consequently the probability becomes lower, which will be undesirable for visualization.) If the system is integrable, the MPMP must be zero dimensional (i.e., points), otherwise one dimensional.

Figure 3 shows the results of the MPMP, where we choose the potential minimum in the first quadrant of the $x_{1} x_{2}$ plane. The MPMP is zero dimensional for $\xi_{0}=0$ [Fig 3(a)], one dimensional for $\xi_{0}=K$ [Fig 3(c)], and intermediate for $\xi_{0}=0.3 K$ [Fig 3(b)], as expected. As we will see in Sec. IV, the MPMP is particularly useful in the quantum case in comparison with the SOS plot. (Although here we focus on 
(a)

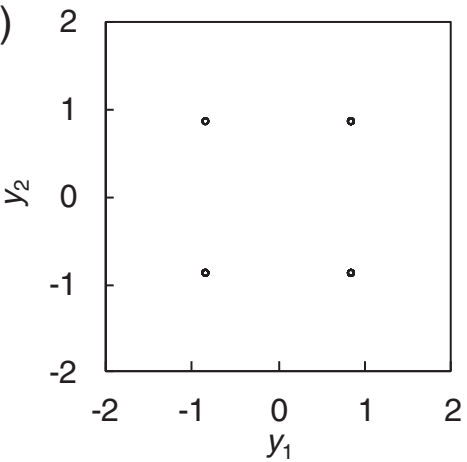

(b)

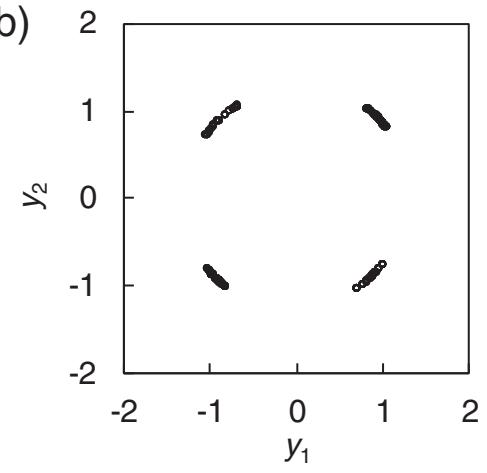

(c)

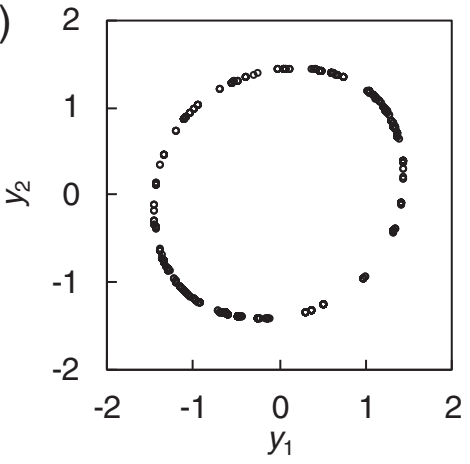

FIG. 3. MPMPs in the classical model given by Eqs. (4)-(6). We choose the potential minimum $\left(X_{1}, X_{2}\right)$ in the first quadrant of the $x_{1} x_{2}$ plane. Circles are obtained by plotting $\left(y_{1}, y_{2}\right)$ when trajectories in numerical simulation pass by close to $\left(X_{1}, X_{2}\right)$. (a) $\xi_{0}=0$ and $\left(X_{1}, X_{2}\right)=(1.73,1.77)$. (b) $\xi_{0}=0.3 \mathrm{~K}$ and $\left(X_{1}, X_{2}\right)=(1.82,1.85)$. (c) $\xi_{0}=K$ and $\left(X_{1}, X_{2}\right)=(2,2.03)$. The other parameters are set as Eqs. (8)-(11). See Appendix B for details.

coupled KPOs, the MPMP approach will be applicable to other systems, such as coupled quartic oscillators [75-78].)

\section{Sensitivity to initial conditions}

Here we also observe the sensitivity to initial conditions in the classical model. The results are shown in Fig. 4. When $\xi_{0}=0$, the Euclidean distance between two trajectories, $\mathbf{x}(t)$ and $\mathbf{x}^{\prime}(t)$, with a very small deviation in their initial conditions saturates at a small value, as shown in Fig. 4(a). This is because in this case, the system is integrable, and then the trajectories from close initial conditions must always be close to each other. In contrast, when $\xi_{0}=0.3 K$ or $K$, the distance exponentially grows, as shown in Figs. 4(b) and 4(c), because of nonintegrability, as expected. (The saturation in the nonintegrable cases comes from the fact that the energetically
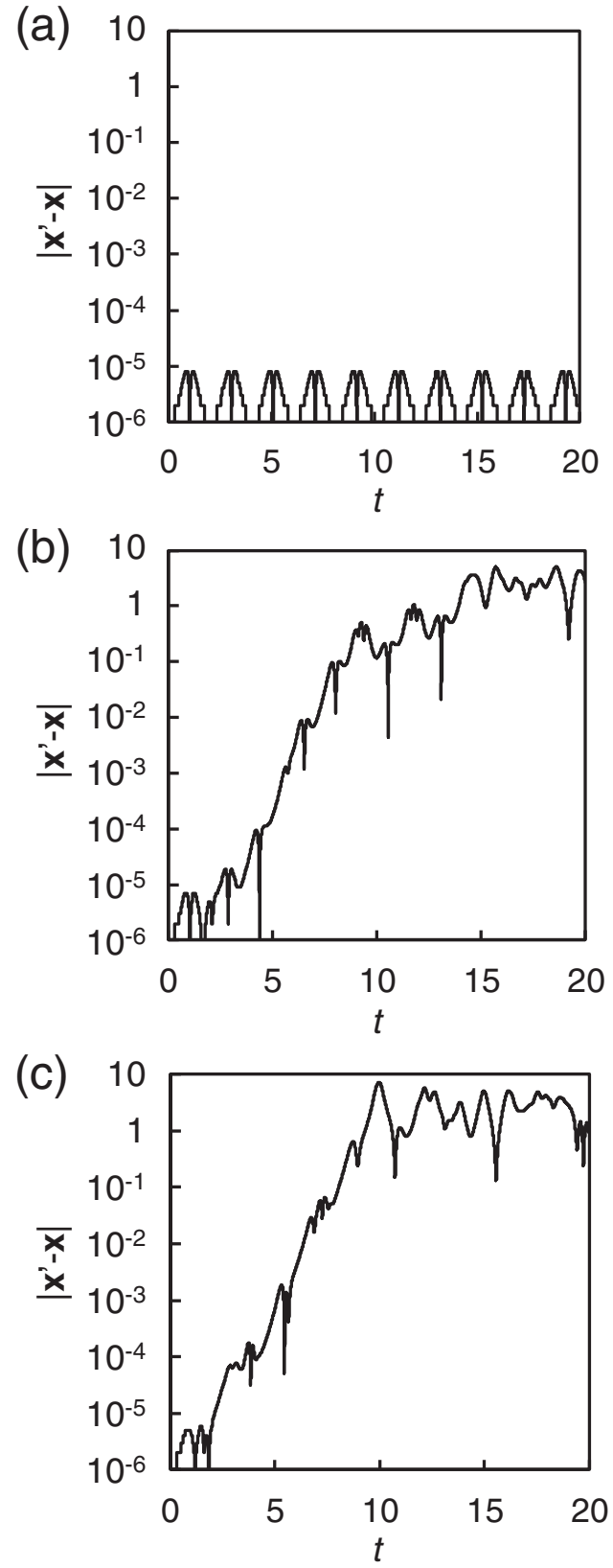

FIG. 4. Initial-condition sensitivity in the classical model given by Eqs. (4)-(6); $\mathbf{x}(t)$ and $\mathbf{x}^{\prime}(t)$ are two trajectories with a very small deviation in their initial conditions. (a) $\xi_{0}=0$. (b) $\xi_{0}=0.3 \mathrm{~K}$. (c) $\xi_{0}=K$. The other parameters are set as Eqs. (8)-(11); $t$ is in unit of $K^{-1}$. See Appendix C for details.

allowable regions are finite.) This together with the above results for SOS and MPMP indicates chaos in the coupled-KPO system.

\section{QUANTUM SIGNATURES OF CHAOS}

\section{A. Quantum SOS plots}

Here we introduce quantum versions of the SOS plot. Instead of plotting intersection points in the classical case, we use the time integral of a quasiprobability distribution, the Wigner function or the Husimi function (also known as the 

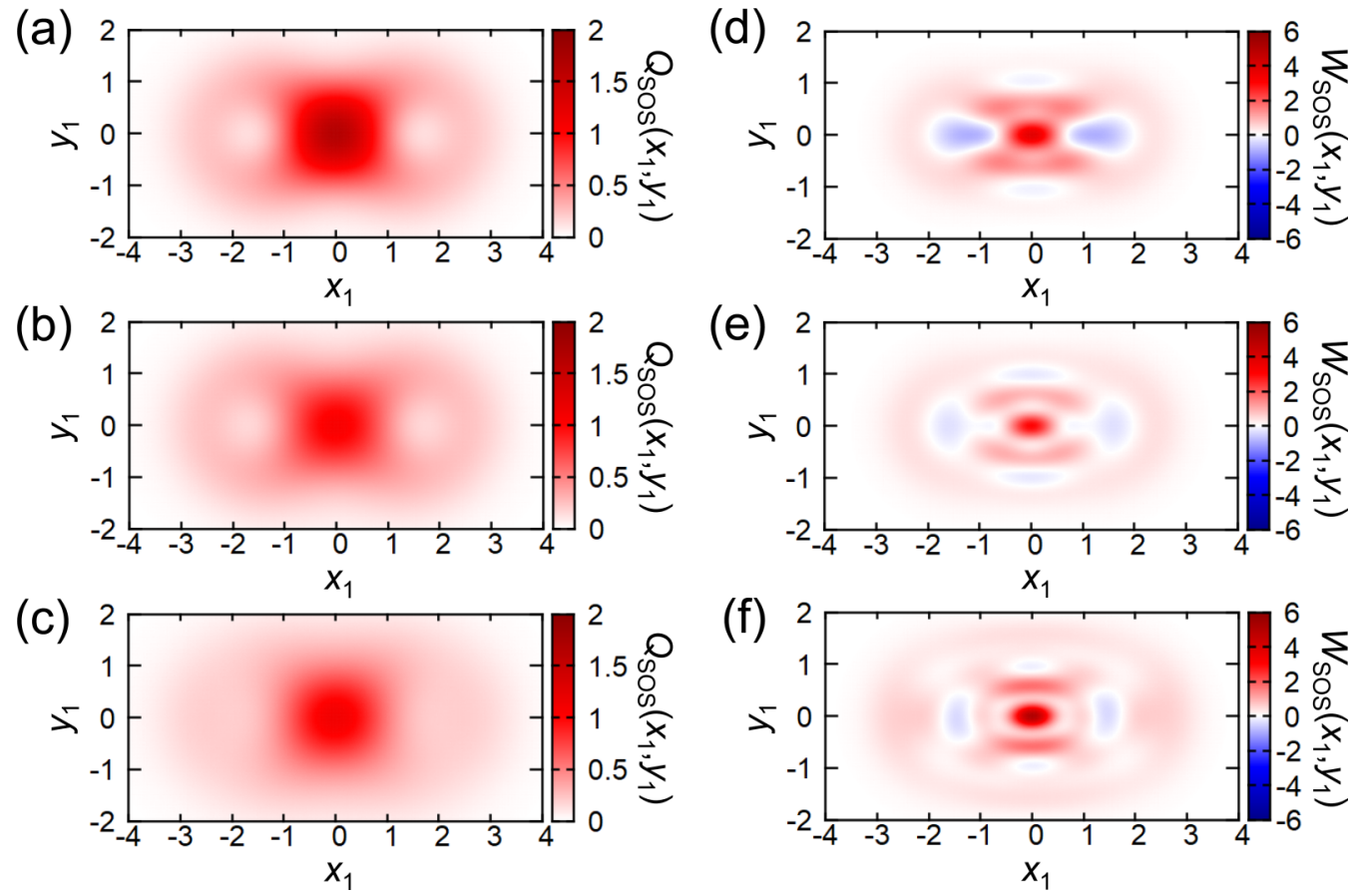

FIG. 5. Quantum SOS plots defined by Eqs. (13) and (14) in the quantum model given by Eqs. (1)-(3). Corresponding classical results are shown in Fig. 2. (a), (d) $\xi_{0}=0$. (b), (e) $\xi_{0}=0.3 K$. (c), (f) $\xi_{0}=K$. The other parameters are set as Eqs. (8)-(11). See Appendix D for details.

Q function) [12,20,51,57], with $y_{2}=0$. Thus we define the quantum versions of the SOS plot as

$$
\begin{aligned}
W_{\mathrm{SOS}}\left(x_{1}, y_{1}\right) & =\int_{0}^{T} d t \int_{-\infty}^{\infty} d x_{2} W\left(x_{1}, x_{2}, y_{1}, 0, t\right), \\
Q_{\mathrm{SOS}}\left(x_{1}, y_{1}\right) & =\int_{0}^{T} d t \int_{-\infty}^{\infty} d x_{2} Q\left(x_{1}, x_{2}, y_{1}, 0, t\right),
\end{aligned}
$$

where $W\left(x_{1}, x_{2}, y_{1}, 0, t\right)$ and $Q\left(x_{1}, x_{2}, y_{1}, 0, t\right)$ are the Wigner and Husimi functions with $y_{2}=0$ for the state vector, $|\psi(t)\rangle$, at time $t$, and $T$ is the final time in each simulation. (A different kind of quantum SOS plot based on the Husimi function was proposed and investigated for a quartic oscillator model [78].)

Figure 5 shows the results of the quantum SOS plots corresponding to the classical ones in Fig. 2. Although the Husimi-type SOS plots shown in Figs. 5(a)-5(c) indicate the classical SOS plots in Figs. 2(a)-2(c) to some extent, it is hard to distinguish integrability (one dimensional) from nonintegrability (two dimensional) because of large quantum fluctuations. (The large fluctuations come from the small mean photon numbers.) The situation is worse in the Wigner case, as shown in Figs. 5(d)-5(f), because of quantum interference. (Similar results have been reported for a single driven pendulum [81].) This is the reason why we have introduced the MPMP in this work.

\section{B. Quantum MPMPs}

Here we introduce quantum versions of the MPMP as

$$
\begin{gathered}
W_{\text {MPMP }}\left(y_{1}, y_{2}\right)=\int_{0}^{T} d t W\left(X_{1}, X_{2}, y_{1}, y_{2}, t\right), \\
Q_{\text {MPMP }}\left(y_{1}, y_{2}\right)=\int_{0}^{T} d t Q\left(X_{1}, X_{2}, y_{1}, y_{2}, t\right),
\end{gathered}
$$

where $\left(X_{1}, X_{2}\right)$ is the position of a minimum of the potential $V_{\mathrm{c}}(\mathbf{x})$ in Eq. (7).

Figure 6 shows the results of the quantum MPMPs together with the corresponding classical results in Fig. 3. The Husimitype MPMPs shown in Figs. 6(a)-6(c) clearly indicate the classical MPMPs even with large quantum fluctuations, which can be regarded as a quantum signature of chaos (nonintegrability). The Wigner-type MPMPs shown in Figs. 6(d)-6(f) also indicate the classical MPMPs to some extent even with quantum interference. Thus the MPMP is particularly useful in quantum cases, in comparison with the SOS plot. (Although here we show the usefulness of the MPMP only for coupled KPOs, this will be useful for other systems, such as coupled quartic oscillators [75-78].)

\section{OTOCs}

Here we discuss the sensitivity to initial conditions in the quantum model. In quantum cases, the initial-condition sensitivity can be evaluated by the OTOCs [58-69] defined by

$$
C_{i, j}(t)=-4\left\langle\psi(0)\left|\left[x_{i}(t), y_{j}(0)\right]^{2}\right| \psi(0)\right\rangle,
$$

where $|\psi(0)\rangle$ is an initial state vector, $\left[O_{1}, O_{2}\right]=O_{1} O_{2}-$ $\mathrm{O}_{2} \mathrm{O}_{1}$ is the commutation relation between two operators, $x_{i}(t)$ is the position operator for the $i$ th KPO at time $t$ in the Heisenberg representation, and $y_{j}(0)$ is the initial momentum operator for the $j$ th KPO. The factor of 4 comes from the definitions of the quadrature amplitudes and their commutation relations:

$$
\begin{aligned}
& x_{i}(0)=\frac{a_{i}+a_{i}^{\dagger}}{2}, \\
& y_{i}(0)=\frac{a_{i}-a_{i}^{\dagger}}{2 i},
\end{aligned}
$$



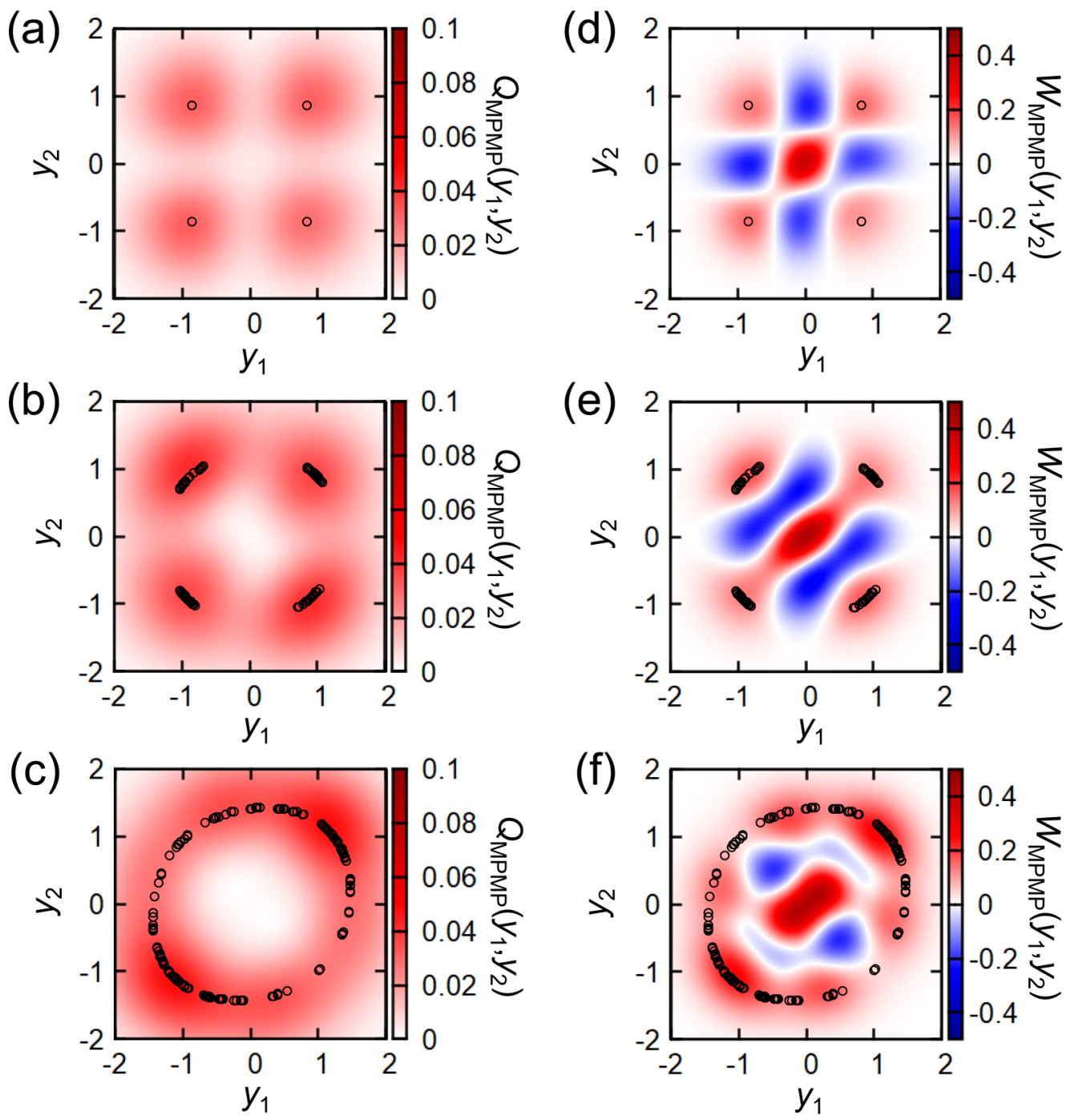

FIG. 6. Quantum MPMPs defined by Eqs. (15) and (16) in the quantum model given by Eqs. (1)-(3). ( $\left.X_{1}, X_{2}\right)$ is set to the position of the potential minimum in the first quadrant of the $x_{1} x_{2}$ plane. Circles represent classical results shown in Fig. 2 . (a), (d) $\xi_{0}=0$ and $\left(X_{1}, X_{2}\right)=(1.73,1.77)$. (b), (e) $\xi_{0}=0.3 K$ and $\left(X_{1}, X_{2}\right)=(1.82,1.85)$. (c), (f) $\xi_{0}=K$ and $\left(X_{1}, X_{2}\right)=(2,2.03)$. The other parameters are set as Eqs. (8)-(11). See Appendix E for details.

$$
\left[x_{i}(0), y_{i}(0)\right]=\frac{i}{2} .
$$

The physical meaning of the OTOCs can be extracted by naively replacing the commutator with the classical Poisson bracket $(i / 2)\left\{x_{i}(t), y_{j}(0)\right\}=(i / 2) \partial x_{i}(t) / \partial x_{j}(0)[58,60]$. That is, the classical counterpart of $C_{i, j}(t)$, which is denoted by $\tilde{C}_{i, j}(t)$, is given by

$$
\tilde{C}_{i, j}(t)=\left\langle\left(\frac{\partial x_{i}(t)}{\partial x_{j}(0)}\right)^{2}\right\rangle,
$$

where $\langle\cdot\rangle$ represents the average over trajectories with different initial conditions related to the quantum initial state. This classical interpretation suggests that the OTOCs are related to the initial-condition sensitivity.

Figure 7 shows the results of the OTOCs, where the solid and dotted lines represent $C_{i, j}(t)$ and $\tilde{C}_{i, j}(t)$, respectively. First of all, the classical results are in good agreement with the quantum results, in particular, around the initial time. This indicates that the above classical interpretation of the OTOCs is valid.

However, unlike the classical initial-condition sensitivity shown in Fig. 4, the OTOCs rapidly increase only around the initial time and soon saturate. This may be due to quantum fluctuations. Here it should be noted that the initial rapid increase of the OTOCs does not indicate chaos because this can be seen even in the integrable case, as shown in Fig. 7(a). Instead, this naturally occurs when the initial state is around an unstable point (maximum of the potential), as discussed recently $[65,66]$.

On the other hand, we can find that the oscillation amplitudes of $C_{1,1}$ in the nonintegrable case $\left(\xi_{0}=0.3 \mathrm{~K}\right.$ and $K$ ) shown in Figs. 7(b) and 7(d) seem smaller than those in the integrable case $\left(\xi_{0}=0\right)$ shown in Fig. 7(a). This difference may be due to more chaotic behavior in the nonintegrable case. This is another quantum signature of chaos proposed recently [62]. (More detailed 

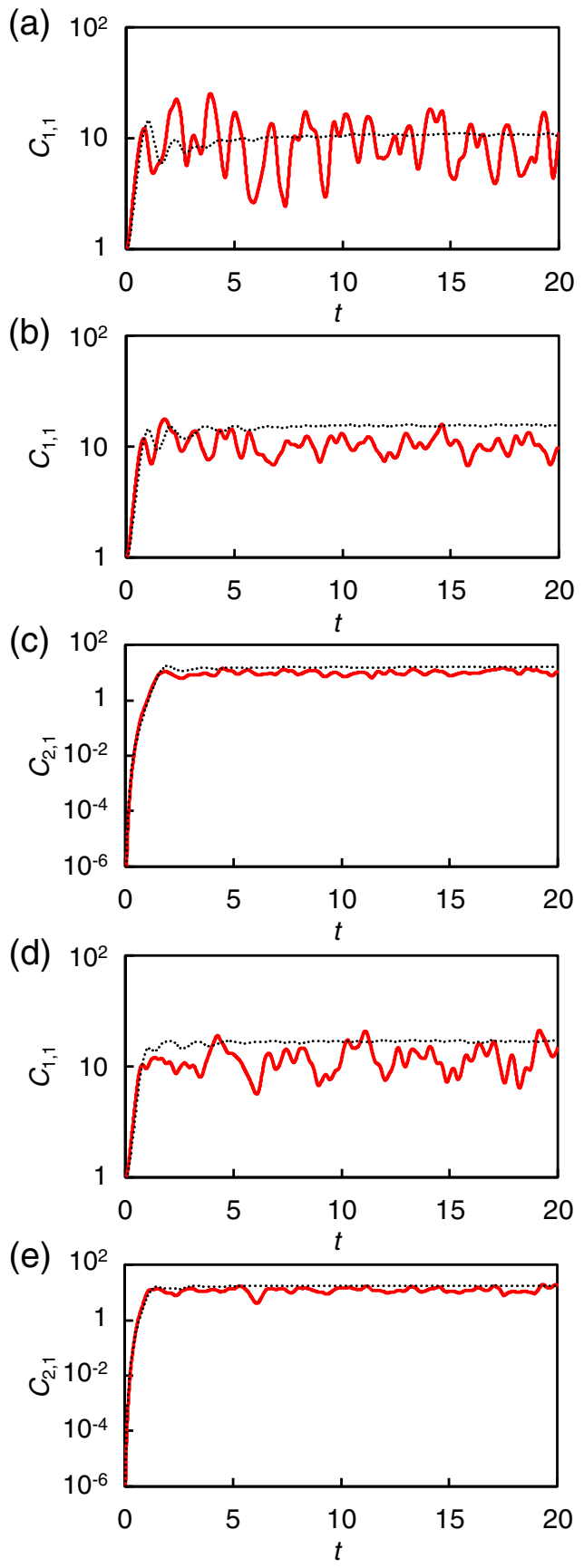

FIG. 7. OTOCs in the quantum model (solid lines) and classical counterparts (dotted lines). (a) $\xi_{0}=0$. (b), (c) $\xi_{0}=0.3 K$. (d), (e) $\xi_{0}=K$. $\left(C_{2,1}\right.$ for $\xi_{0}=0$ is not shown because in the decoupled case, $C_{2,1}$ is exactly zero.) The other parameters are set as Eqs. (8)-(11); $t$ is in unit of $K^{-1}$. See Appendix $\mathrm{F}$ for details.

studies using OTOCs are desirable, but left for future work.)

\section{Energy-level spacing statistics}

Finally, we check a conventional quantum signature of chaos: Energy-level spacing statistics [51,70-73]. (There are
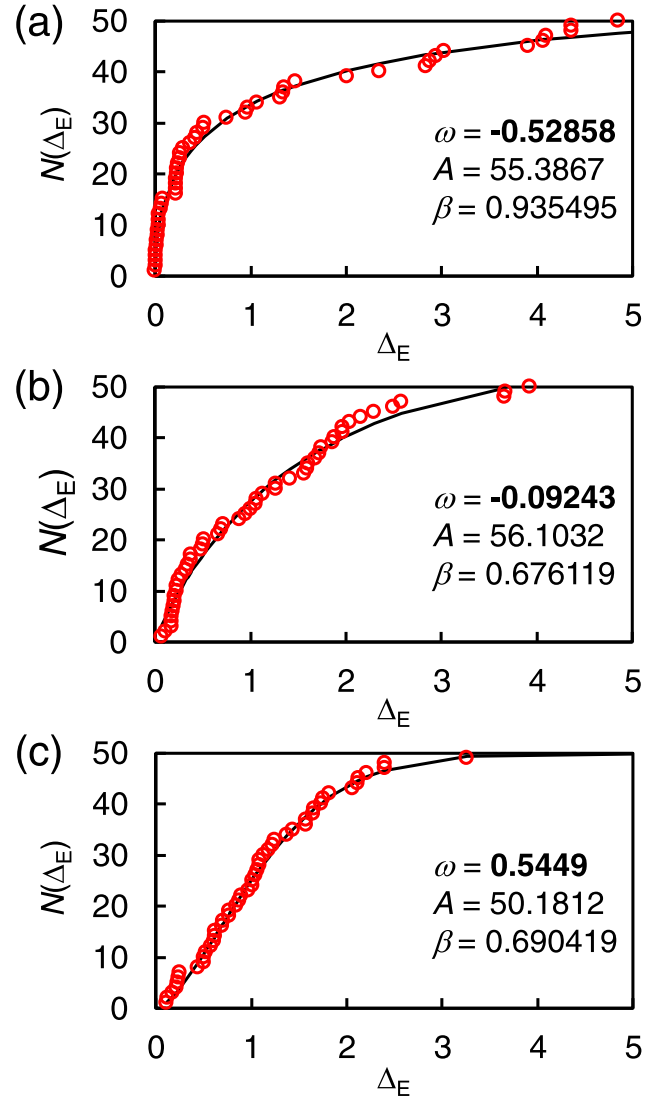

FIG. 8. Cumulative energy-level spacing distributions in the quantum model given by Eqs. (1)-(3). $N\left(\Delta_{\mathrm{E}}\right)$ denotes the number of level spacing equal to or smaller than $\Delta_{\mathrm{E}}$. Curves and three parameters $(\omega, A$, and $\beta)$ are fitting results using the function form $A\left(1-e^{-\beta \Delta_{\mathrm{E}}^{\omega+1}}\right.$ ). (a) $\xi_{0}=0$. (b) $\xi_{0}=0.3 K$. (c) $\xi_{0}=K$. The other parameters are set as Eqs. (8)-(11). See Appendix G for details.

more approaches to quantum chaos using energy eigenvalues [76,82-87] and eigenstates [75,77,88,89]. However, here we focus on the most conventional approach and leave other ones for future work.) It is known that the energy-level spacing $\Delta_{\mathrm{E}}$ defined by the difference between two neighboring energy levels obeys the Poisson distribution $\left(\alpha e^{-\beta \Delta_{\mathrm{E}}}\right)$ in the integrable (regular) case and the Wigner distribution $\left(\alpha \Delta_{\mathrm{E}} e^{-\beta \Delta_{\mathrm{E}}^{2}}\right)$ in the nonintegrable (chaotic) case ( $\beta$ is a constant). This means that the probability for zero spacing decreases as the system becomes more chaotic, which is due to avoided crossings of energy levels induced by complex interactions in chaotic systems [51].

The two distributions are unified as $\Delta_{\mathrm{E}}^{\omega} e^{-\beta \Delta_{\mathrm{E}}^{\omega+1}}[70,71]$, where $\omega=0$ and 1 correspond to Poisson and Wigner, respectively. Note that this distribution can be integrated analytically, which leads to the cumulative level spacing distribution $\propto\left(1-e^{-\beta \Delta_{\mathrm{E}}^{\omega+1}}\right)$. Figure 8 shows the cumulative distributions of the present quantum model together with fitting curves using the function form $A\left(1-e^{-\beta \Delta_{\mathrm{E}}^{\omega+1}}\right)$ and the fitting results of the three parameters. The exponent $\omega$ is larger for stronger coupling and exceeds 0.5 when $\xi_{0}=K$. This is another quantum signature of chaos in the coupled-KPO system. 


\section{CONCLUSIONS}

We have investigated the quantum and classical models for two coupled nondissipative KPOs from the viewpoint of chaos. Using the Poincaré SOS plot, the MPMP (a lowerdimensional version of the SOS plot), and the initial-condition sensitivity, we have shown that the classical model with nonzero coupling is nonintegrable and hence exhibits chaotic behavior. We have also provided quantum signatures of chaos using quantum versions of the SOS plot and the MPMP defined with time integrals of the Wigner and Husimi functions, OTOCs, and energy-level spacing statistics. We have found that the quantum MPMP can distinguish integrability and nonintegrability clearly even at a few-photon level (more clearly than the quantum SOS plot), and also that the long-term behavior, not the initial behavior, of the OTOCs can be regarded as a quantum signature of chaos. The present results suggest that the system of coupled KPOs will be useful not only for quantum computing but also for the study on quantum chaos. We also expect that such an understanding of the nonlinear dynamics in the KPO network will be useful for its applications, such as quantum computing and quantum-inspired algorithms [56].

\section{ACKNOWLEDGMENT}

This work was supported by JST ERATO (Grant No. JPMJER1601).

\section{APPENDIX A: SOS PLOT IN THE CLASSICAL MODEL}

To obtain the SOS plots in Fig. 2, we numerically solve Eqs. (4)-(6) by the fourth-order Runge-Kutta method with a time step of $\Delta_{t}=10^{-4}$ from $t=0$ to 20 . The initial conditions are set as $x_{1}(0)=x_{2}(0)=0$ and $y_{i}(0)=10^{-6} r_{i}$, where $r_{1}$ and $r_{2}$ are independent random numbers from the standard normal distribution. We plot $\left(x_{1}, y_{1}\right)$ when $y_{2}(t) y_{2}\left(t-\Delta_{t}\right)<0$, which means that the trajectory have crossed the plane $y_{2}=0$. We iterated the simulation 200 times to obtain enough points.

\section{APPENDIX B: MPMP IN THE CLASSICAL MODEL}

To obtain the MPMPs in Fig. 3, we did the same simulation as in the SOS case explained in Appendix A. We plot $\left(y_{1}, y_{2}\right)$ when $|\mathbf{x}-\mathbf{X}|<10^{-3}$, where $\mathbf{X}$ denotes the position of the potential minimum in the first quadrant of the $x_{1} x_{2}$ plane. $\mathbf{X}$ is numerically found as $\mathbf{X}=(1.73,1.77)$ for $\xi_{0}=0, \mathbf{X}=(1.82,1.85)$ for $\xi_{0}=0.3 K$, and $\mathbf{X}=(2,2.03)$ for $\xi_{0}=K$. We iterated the simulation $10^{5}$ times to obtain enough points.

\section{APPENDIX C: INITIAL-CONDITION SENSITIVITY IN THE CLASSICAL MODEL}

To obtain Fig. 4, we did the same simulation as above with the following initial conditions (no iteration):

$$
\begin{gathered}
x_{1}(0)=x_{2}(0)=x_{2}^{\prime}(0)=0, \\
x_{1}^{\prime}(0)=10^{-6}, \\
y_{1}(0)=y_{1}^{\prime}(0)=0.5 \cos (0.65 \pi), \\
y_{2}(0)=y_{2}^{\prime}(0)=0.5 \sin (0.65 \pi) .
\end{gathered}
$$

\section{APPENDIX D: QUANTUM SOS PLOTS}

Here we give the formulations of $W_{\mathrm{SOS}}\left(x_{1}, y_{1}\right)$ and $Q_{\text {SOS }}\left(x_{1}, y_{1}\right)$ defined by Eqs. (13) and (14) in the photonnumber basis.

First of all, the Wigner and Husimi functions are defined as [20,57]

$$
\begin{gathered}
W(\mathbf{x}, \mathbf{y}, t)=\left(\frac{2}{\pi}\right)^{2} \operatorname{tr}\left[D^{(1)}\left(2 \alpha_{1}\right) P_{1} D^{(2)}\left(2 \alpha_{2}\right) P_{2} \rho(t)\right], \\
Q(\mathbf{x}, \mathbf{y}, t)=\frac{1}{\pi^{2}}\left\langle\alpha_{1}\left|\left\langle\alpha_{2}|\rho(t)| \alpha_{1}\right\rangle\right| \alpha_{2}\right\rangle,
\end{gathered}
$$

where $\alpha_{i}=x_{i}+i y_{i}, \quad D^{(i)}(\alpha)=e^{\alpha a_{i}^{\dagger}-\alpha^{*} a_{i}}$ is the so-called displacement operator, $P_{i}=e^{i a_{i}^{\dagger} a_{i}}$ is the parity operator, $\left|\alpha_{i}\right\rangle=D^{(i)}\left(\alpha_{i}\right)|0\rangle$ is a coherent state $(|0\rangle$ is the vacuum state), and $\rho(t)=|\psi(t)\rangle\langle\psi(t)|$ is the density operator corresponding to the state vector $|\psi(t)\rangle$.

In the photon-number basis $\left\{\left|n_{1}\right\rangle\left|n_{2}\right\rangle\right\}$, we obtain [20]

$$
\begin{gathered}
W(\mathbf{x}, \mathbf{y}, t)=\left(\frac{2}{\pi}\right)^{2} \sum_{m_{1}=0}^{\infty} \sum_{m_{2}=0}^{\infty} \sum_{n_{1}=0}^{\infty} \sum_{n_{2}=0}^{\infty}(-1)^{n_{1}+n_{2}} D_{m_{1}, n_{1}}^{(1)}\left(2 \alpha_{1}\right) D_{m_{2}, n_{2}}^{(2)}\left(2 \alpha_{2}\right) \psi_{n_{1}, n_{2}}(t) \psi_{m_{1}, m_{2}}^{*}(t), \\
Q(\mathbf{x}, \mathbf{y}, t)=\frac{1}{\pi^{2}} \sum_{m_{1}=0}^{\infty} \sum_{m_{2}=0}^{\infty} \sum_{n_{1}=0}^{\infty} \sum_{n_{2}=0}^{\infty}\left\langle\alpha_{1} \mid n_{1}\right\rangle\left\langle\alpha_{2} \mid n_{2}\right\rangle \psi_{n_{1}, n_{2}}(t) \psi_{m_{1}, m_{2}}^{*}(t)\left\langle m_{1} \mid \alpha_{1}\right\rangle\left\langle m_{2} \mid \alpha_{2}\right\rangle,
\end{gathered}
$$

with

$$
\begin{gathered}
\psi_{n_{1}, n_{2}}(t)=\left\langle n_{1}\right|\left\langle n_{2} \mid \psi(t)\right\rangle \\
D_{m_{i}, n_{i}}^{(i)}(\alpha)=e^{-|\alpha|^{2} / 2} \sqrt{m_{i} ! n_{i} !} \sum_{k=0}^{\min \left(m_{i}, n_{i}\right)} \frac{1}{k !} \frac{\alpha^{m_{i}-k}}{\left(m_{i}-k\right) !} \frac{\left(-\alpha^{*}\right)^{n_{i}-k}}{\left(n_{i}-k\right) !} \\
\left\langle n_{i} \mid \alpha_{i}\right\rangle=\frac{\alpha_{i}^{n_{i}}}{\sqrt{n_{i} !}} e^{-\left|\alpha_{i}\right|^{2} / 2} .
\end{gathered}
$$


The integrals $\int_{-\infty}^{\infty} d x_{2} W\left(x_{1}, x_{2}, y_{1}, 0, t\right)$ and $\int_{-\infty}^{\infty} d x_{2} Q\left(x_{1}, x_{2}, y_{1}, 0, t\right)$ required for $W_{\mathrm{SOS}}\left(x_{1}, y_{1}\right)$ and $Q_{\mathrm{SOS}}\left(x_{1}, y_{1}\right)$ are obtained by Eqs. (D3)-(D7) together with the following formulas:

$$
\begin{gathered}
\int_{-\infty}^{\infty} d x_{2} D_{m_{2}, n_{2}}^{(2)}\left(2 x_{2}\right)=\sqrt{\frac{\pi}{2} m_{2} ! n_{2} !} \sum_{k=0}^{\min \left(m_{2}, n_{2}\right)} \frac{(-1)^{n_{2}-k}\left(m_{2}+n_{2}-2 k-1\right) ! !}{k !\left(m_{2}-k\right) !\left(n_{2}-k\right) !} \delta_{\text {even }}\left(m_{2}+n_{2}\right) \\
\int_{-\infty}^{\infty} d x_{2}\left\langle\alpha_{2} \mid m_{2}\right\rangle\left\langle n_{2} \mid \alpha_{2}\right\rangle=\sqrt{\frac{\pi}{m_{2} ! n_{2} !} \frac{\left(m_{2}+n_{2}-1\right) ! !}{2^{\left(m_{2}+n_{2}\right) / 2}} \delta_{\text {even }}\left(m_{2}+n_{2}\right)}
\end{gathered}
$$

where $\delta_{\text {even }}(n)=1$ if $n$ is even, otherwise $\delta_{\text {even }}(n)=0$.

The numerical results of $W_{\mathrm{SOS}}\left(x_{1}, y_{1}\right)$ and $Q_{\mathrm{SOS}}\left(x_{1}, y_{1}\right)$ shown in Fig. 5 are obtained by accumulating the integrals $\int_{-\infty}^{\infty} d x_{2} W\left(x_{1}, x_{2}, y_{1}, 0, t\right)$ and $\int_{-\infty}^{\infty} d x_{2} Q\left(x_{1}, x_{2}, y_{1}, 0, t\right)$ multiplied by a time step of $\Delta_{t}=10^{-3}$ from $t=0$ to $T=20$. Here $\psi_{n_{1}, n_{2}}(t)$ necessary for the integrals is obtained by solving the Schrödinger equation with the Hamiltonian in Eq. (1) in the photon-number basis by the fourth-order Runge-Kutta method with the time step of $\Delta_{t}=10^{-3}$, the initial state set to the vacuum state, and the maximum photon number of 30 .

\section{APPENDIX E: QUANTUM MPMPS}

The numerical results of $W_{\mathrm{MPMP}}\left(y_{1}, y_{2}\right)$ and $Q_{\mathrm{MPMP}}\left(y_{1}, y_{2}\right)$ shown in Fig. 6 are obtained by accumulating the Wigner and
Husimi functions multiplied by a time step of $\Delta_{t}=10^{-3}$ from $t=0$ to $T=20$, where the Wigner and Husimi functions are obtained by using Eqs. (D3)-(D7) with $\psi_{n_{1}, n_{2}}(t)$ obtained by the same simulation as in Appendix D. $\left(X_{1}, X_{2}\right)$ is numerically found, as mentioned in Appendix B.

\section{APPENDIX F: OTOC}

The results shown in Fig. 7 are obtained as follows.

Using $x(t)=e^{i H t} x(0) e^{-i H t}$, the OTOC defined by Eq. (17) is formulated in the eigenenergy basis $\left\{\left|E_{k}\right\rangle\right\}$ as [60]

$$
\begin{gathered}
C_{i, j}(t)=-4 \sum_{k=0}^{\infty} \sum_{m=0}^{\infty} \sum_{n=0}^{\infty}\left\langle\psi(0) \mid E_{k}\right\rangle\left\langle E_{k}\left|\left[x_{i}(t), y_{j}(0)\right]\right| E_{m}\right\rangle\left\langle E_{m}\left|\left[x_{i}(t), y_{j}(0)\right]\right| E_{n}\right\rangle\left\langle E_{n} \mid \psi(0)\right\rangle, \\
\left\langle E_{k}\left|\left[x_{i}(t), y_{j}(0)\right]\right| E_{m}\right\rangle=\sum_{l=0}^{\infty}\left[e^{i\left(E_{k}-E_{l}\right) t}\left\langle E_{k}\left|x_{i}(0)\right| E_{l}\right\rangle\left\langle E_{l}\left|y_{j}(0)\right| E_{m}\right\rangle-e^{i\left(E_{l}-E_{m}\right) t}\left\langle E_{k}\left|y_{j}(0)\right| E_{l}\right\rangle\left\langle E_{l}\left|x_{i}(0)\right| E_{m}\right\rangle\right] .
\end{gathered}
$$

Thus we can obtain $C_{i, j}(t)$ by using $\left\{\left|E_{k}\right\rangle\right\}$ obtained by numerically diagonalizing the Hamiltonian in the photon-number basis with the maximum photon number of 30 , the same as the above simulation, and using the definitions of $x_{i}(0)$ and $y_{i}(0)$ in Eqs. (18) and (19). For the comparison with the results in Fig. 4, the initial state is set to coherent states as $|\psi(0)\rangle=\left|\alpha_{1}^{(0)}\right\rangle\left|\alpha_{2}^{(0)}\right\rangle$, where $\alpha_{1}^{(0)}=x_{1}^{(0)}+i y_{1}^{(0)}\left[x_{1}^{(0)}=0\right.$ and $\left.y_{1}^{(0)}=0.5 \cos (0.65 \pi)\right]$ and $\alpha_{2}^{(0)}=x_{2}^{(0)}+i y_{2}^{(0)}\left[x_{2}^{(0)}=0\right.$ and $\left.y_{2}^{(0)}=0.5 \sin (0.65 \pi)\right]$, which correspond to the initial conditions for Fig. 4 in Eqs. (C1)-(C4). The resultant $C_{i, j}(t)$ are shown by the solid lines in Fig. 7.

For the classical counterparts, we calculate two trajectories, $\mathbf{x}(t)$ and $\mathbf{x}^{\prime}(t)$, with the following initial conditions:

$$
\begin{gathered}
x_{1}(0)=x_{1}^{(0)}+\Delta_{x} r_{1}, \\
x_{1}^{\prime}(0)=x_{1}(0)+\delta_{x}, \\
x_{2}(0)=x_{2}^{\prime}(0)=x_{2}^{(0)}+\Delta_{x} r_{2}, \\
y_{1}(0)=y_{1}^{\prime}(0)=y_{1}^{(0)}+\Delta_{y} r_{3}, \\
y_{2}(0)=y_{2}^{\prime}(0)=y_{2}^{(0)}+\Delta_{y} r_{4},
\end{gathered}
$$

where $r_{j}(j=1, \ldots, 4)$ are independent random numbers from the standard normal distribution, $\Delta_{x}=\Delta_{y}=0.5$ correspond to the standard deviations of the quantum fluctuations of $x_{i}$ and $y_{i}$, and $\delta_{x}=0.5$ is the deviation for the evaluation of the partial derivative in Eq. (21). (The large $\delta_{x}$ comparable to the quantum fluctuations is used to mimic the saturation of the OTOCs in the quantum model.) Using the two trajectories, $\tilde{C}_{i, 1}(t)$ shown by the dotted lines in Fig. 7 are obtained as follows:

$$
\tilde{C}_{i, 1}(t)=\left\langle\left(\frac{x_{i}^{\prime}(t)-x_{i}(t)}{\delta_{x}}\right)^{2}\right\rangle,
$$

where the average was taken over $10^{4}$ iterations.

\section{APPENDIX G: ENERGY-LEVEL SPACING STATISTICS}

The results in Fig. 8 are obtained as follows. First we numerically diagonalize the Hamiltonian in Eq. (1) in the photon-number basis with the maximum photon number of 30 , the same as above. Then, taking the parity invariance of the Hamiltonian into account, we classify the energy eigenstates into two groups with even and odd total photon numbers. Here we focus on the even eigenstates and sort the corresponding energies in ascending order. Thus we obtain the energy-level spacing as the difference between two neighboring energies. To avoid the effects of the finite photon numbers, we take 50 spacings from the smallest, which are plotted in Fig. 8. The curves in Fig. 8 are obtained by fitting $A\left(1-e^{-\beta \Delta_{\mathrm{E}}^{\omega+1}}\right)$ to the 50 points, where $\omega, A$, and $\beta$ are fitting parameters. 
[1] M. A. Nielsen and I. L. Chuang, Quantum Computation and Quantum Information (Cambridge University Press, Cambridge, 2000).

[2] D. Gottesman, A. Kitaev, and J. Preskill, Encoding a qubit in an oscillator, Phys. Rev. A 64, 012310 (2001).

[3] P. T. Cochrane, G. J. Milburn, and W. J. Munro, Macroscopically distinct quantum-superposition states as a bosonic code for amplitude damping, Phys. Rev. A 59, 2631 (1999).

[4] M. H. Michael, M. Silveri, R. T. Brierley, V. V. Albert, J. Salmilehto, L. Jiang, and S. M. Girvin, New Class of Quantum Error-Correcting Codes for a Bosonic Mode, Phys. Rev. X 6, 031006 (2016).

[5] N. Ofek, A. Petrenko, R. Heeres, P. Reinhold, Z. Leghtas, B. Vlastakis, Y. Liu, L. Frunzio, S. M. Girvin, L. Jiang, M. Mirrahimi, M. H. Devoret, and R. J. Schoelkopf, Extending the lifetime of a quantum bit with error correction in superconducting circuits, Nature (London) 536, 441 (2016).

[6] K. S. Chou, J. Z. Blumoff, C. S. Wang, P. C. Reinhold, C. J. Axline, Y. Y. Gao, L. Frunzio, M. H. Devoret, L. Jiang, and R. J. Schoelkopf, Deterministic teleportation of a quantum gate between two logical qubits, Nature (London) 561, 368 (2018).

[7] L. Hu, Y. Ma, W. Cai, X. Mu, Y. Xu, W. Wang, Y. Wu, H. Wang, Y. P. Song, C.-L. Zou, S. M. Girvin, L-M. Duan, and L. Sun, Quantum error correction and universal gate set operation on a binomial bosonic logical qubit, Nat. Phys. 15, 503 (2019).

[8] C. Flühmann, T. L. Nguyen, M. Marinelli, V. Negnevitsky, K. Mehta, and J. P. Home, Encoding a qubit in a trapped-ion mechanical oscillator, Nature (London) 566, 513 (2019).

[9] P. Campagne-Ibarcq, A. Eickbusch, S. Touzard, E. ZalysGeller, N. E. Frattini, V. V. Sivak, P. Reinhold, S. Puri, S. Shankar, R. J. Schoelkopf, L. Frunzio, M. Mirrahimi, and M. H. Devoret, Quantum error correction of a qubit encoded in grid states of an oscillator, Nature (London) 584, 368 (2020).

[10] M. Mirrahimi, Z. Leghtas, V. V Albert, S. Touzard, R. J Schoelkopf, L. Jiang, and M. H Devoret, Dynamically protected cat-qubits: A new paradigm for universal quantum computation, New. J. Phys. 16, 045014 (2014).

[11] V. V. Albert, C. Shu, S. Krastanov, C. Shen, R.-B. Liu, Z.-B. Yang, R. J. Schoelkopf, M. Mirrahimi, M. H. Devoret, and L. Jiang, Holonomic Quantum Control with Continuous Variable Systems, Phys. Rev. Lett. 116, 140502 (2016).

[12] D. F. Walls and G. J. Milburn, Quantum Optics (Springer, Berlin, 1994).

[13] Z. Leghtas, S. Touzard, I. M. Pop, A. Kou, B. Vlastakis, A. Petrenko, K. M. Sliwa, A. Narla, S. Shankar, M. J. Hatridge, M. Reagor, L. Frunzio, R. J. Schoelkopf, M. Mirrahimi, and M. H. Devoret, Confining the state of light to a quantum manifold by engineered two-photon loss, Science 347, 853 (2015).

[14] S. Touzard, A. Grimm, Z. Leghtas, S. O. Mundhada, P. Reinhold, C. Axline, M. Reagor, K. Chou, J. Blumoff, K. M. Sliwa, S. Shankar, L. Frunzio, R. J. Schoelkopf, M. Mirrahimi, and M. H. Devoret, Coherent Oscillations Inside a Quantum Manifold Stabilized by Dissipation, Phys. Rev. X 8, 021005 (2018).

[15] R. Lescanne, M. Villiers, T. Peronnin, A. Sarlette, M. Delbecq, B. Huard, T. Kontos, M. Mirrahimi, and Z. Leghtas, Exponential suppression of bit-flips in a qubit encoded in an oscillator, Nat. Phys. 16, 509 (2020).
[16] J. Guillaud and M. Mirrahimi, Repetition Cat Qubits for Fault-Tolerant Quantum Computation, Phys. Rev. X 9, 041053 (2019).

[17] C. Chamberland, K. Noh, P. Arrangoiz-Arriola, E. T. Campbell, C. T. Hann, J. Iverson, H. Putterman, T. C. Bohdanowicz, S. T. Flammia, A. Keller, G. Refael, J. Preskill, L. Jiang, A. H. Safavi-Naeini, O. Painter, and F. G. S. L. Brandão, Building a fault-tolerant quantum computer using concatenated cat codes, arXiv:2012.04108.

[18] G. J. Milburn and C. A. Holmes, Quantum coherence and classical chaos in a pulsed parametric oscillator with a Kerr nonlinearity, Phys. Rev. A 44, 4704 (1991).

[19] B. Wielinga and G. J. Milburn, Quantum tunneling in a Kerr medium with parametric pumping, Phys. Rev. A 48, 2494 (1993).

[20] H. Goto, Bifurcation-based adiabatic quantum computation with a nonlinear oscillator network, Sci. Rep. 6, 21686 (2016).

[21] H. Goto, Quantum computation based on quantum adiabatic bifurcations of Kerr-nonlinear parametric oscillators, J. Phys. Soc. Jpn. 88, 061015 (2019).

[22] Z. Wang, M. Pechal, E. A. Wollack, P. Arrangoiz-Arriola, M Gao, N. R. Lee, and A. H. Safavi-Naeini, Quantum Dynamics of a Few-Photon Parametric Oscillator, Phys. Rev. X 9, 021049 (2019).

[23] A. Grimm, N. E. Frattini, S. Puri, S. O. Mundhada, S. Touzard, M. Mirrahimi, S. M. Girvin, S. Shankar, and M. H. Devoret, Stabilization and operation of a Kerr-cat qubit, Nature (London) 584, 205 (2020).

[24] T. Yamaji, S. Kagami, A. Yamaguchi, T. Satoh, K. Koshino, H. Goto, Z. R. Lin, Y. Nakamura, and T. Yamamoto, Spectroscopic observation of crossover from classical Duffing oscillator to Kerr parametric oscillator, arXiv:2010.02621.

[25] E. Farhi, J. Goldstone, S. Gutmann, and M. Sipser, Quantum computation by adiabatic evolution, arXiv:quant-ph/0001106.

[26] E. Farhi, J. Goldstone, S. Gutmann, J. Lapan, A. Lundgren, and D. Preda, A Quantum adiabatic evolution algorithm applied to random instances of an NP-complete problem, Science 292, 472 (2001).

[27] T. Albash and D. A. Lidar, Adiabatic quantum computation, Rev. Mod. Phys. 90, 015002 (2018).

[28] T. Kadowaki and H. Nishimori, Quantum annealing in the transverse Ising model, Phys. Rev. E 58, 5355 (1998).

[29] A. Das and B. K. Chakrabarti, Colloquium: Quantum annealing and analog quantum computation, Rev. Mod. Phys. 80, 1061 (2008).

[30] S. E. Nigg, N. Lörch, and R. P. Tiwari, Robust quantum optimizer with full connectivity, Sci. Adv. 3, e1602273 (2017).

[31] S. Puri, C. K. Andersen, A. L. Grimsmo, and A. Blais, Quantum annealing with all-to-all connected nonlinear oscillators, Nat. Commun. 8, 15785 (2017).

[32] P. Zhao, Z. Jin, P. Xu, X. Tan, H. Yu, and Y. Yu, Two-Photon Driven Kerr Resonator for Quantum Annealing with ThreeDimensional Circuit QED, Phys. Rev. Appl. 10, 024019 (2018).

[33] T. Onodera, E. Ng, and P. L. McMahon, A quantum annealer with fully programmable all-to-all coupling via Floquet engineering, npj Quantum Inf. 6, 48 (2020).

[34] H. Goto and T. Kanao, Quantum annealing using vacuum states as effective excited states of driven systems, Commun. Phys. $\mathbf{3}$ 235 (2020). 
[35] T. Kanao and H. Goto, High-accuracy Ising machine using Kerr-nonlinear parametric oscillators with local four-body interactions, npj Quantum Inf. 7, 18 (2021).

[36] H. Goto, Universal quantum computation with a nonlinear oscillator network, Phys. Rev. A 93, 050301(R) (2016).

[37] S. Puri, S. Boutin, and A. Blais, Engineering the quantum states of light in a Kerr-nonlinear resonator by two-photon driving, npj Quantum Inf. 3, 18 (2017).

[38] S. Puri, L. St-Jean, J. A. Gross, A. Grimm, N. E. Frattini, P. S. Iyer, A. Krishna, S. Touzard, L. Jiang, A. Blais, S. T. Flammia, and S. M. Girvin, Bias-preserving gates with stabilized cat qubits, Sci. Adv. 6, eaay5901 (2020).

[39] T. Kanao, S. Masuda, S. Kawabata, and H. Goto, Quantum gate for Kerr-nonlinear parametric oscillator using effective excited states, arXiv:2108.03091.

[40] Q. Xu, J. K. Iverson, F. G. S. L. Brandao, and L. Jiang, Engineering fast bias-preserving gates on stabilized cat qubits, arXiv:2105.13908.

[41] S. Puri, A. Grimm, P. Campagne-Ibarcq, A. Eickbusch, K. Noh, G. Roberts, L. Jiang, M. Mirrahimi, M. H. Devoret, and S. M. Girvin, Stabilized Cat in a Driven Nonlinear Cavity: A FaultTolerant Error Syndrome Detector, Phys. Rev. X 9, 041009 (2019).

[42] A. S. Darmawan, B. J. Brown, A. L. Grimsmo, D. K. Tuckett, and S. Puri, Practical quantum error correction with the XZZX code and Kerr-cat qubits, PRX Quantum 2, 030345 (2021).

[43] H. Goto, Z. R. Lin, T. Yamamoto, and Y. Nakamura, Ondemand generation of traveling cat states using a parametric oscillator, Phys. Rev. A 99, 023838 (2019).

[44] I. Strandberg, G. Johansson, and F. Quijandría, Wigner negativity in the steady-state output of a Kerr parametric oscillator, Phys. Rev. Research 3, 023041 (2021).

[45] H. Goto, Z. Lin, and Y. Nakamura, Boltzmann sampling from the Ising model using quantum heating of coupled nonlinear oscillators, Sci. Rep. 8, 7154 (2018).

[46] M. Mamaev, L. C. G. Govia, and A. A. Clerk, Dissipative stabilization of entangled cat states using a driven Bose-Hubbard dimer, Quantum 2, 58 (2018).

[47] M. Kewming, S. Shrapnel, and G. Milburn, Quantum correlations in the Kerr Ising model, New J. Phys. 22, 053042 (2020).

[48] V. Savona, Spontaneous symmetry breaking in a quadratically driven nonlinear photonic lattice, Phys. Rev. A 96, 033826 (2017).

[49] R. Rota, F. Minganti, C. Ciuti, and V. Savona, Quantum Critical Regime in a Quadratically Driven Nonlinear Photonic Lattice, Phys. Rev. Lett. 122, 110405 (2019).

[50] S. H. Strogatz, Nonlinear Dynamics and Chaos: With Applications to Physics, Biology, Chemistry, and Engineering, ed. 2 (Westview Press, Boulder, CO, USA, 2015).

[51] S. Wimberger, Nonlinear Dynamics and Quantum Chaos (Springer, Cham, 2014).

[52] Z. Wang, A. Marandi, K. Wen, R. L. Byer, and Y. Yamamoto, Coherent Ising machine based on degenerate optical parametric oscillators, Phys. Rev. A 88, 063853 (2013).

[53] C. Gardiner and P. Zoller, Quantum Noise (Springer-Verlarg, Berlin, 2000).

[54] H.-P. Breuer and F. Petruccione, The Theory of Open Quantum Systems (Oxford University Press, Oxford, 2002).

[55] G. H. Hovsepyan, A. R. Shahinyan, L. Y. Chew, and G. Y. Kryuchkyan, Phase locking and quantum statistics in a para- metrically driven nonlinear resonator, Phys. Rev. A 93, 043856 (2016).

[56] H. Goto, K. Tatsumura, and A. R. Dixon, Combinatorial optimization by simulating adiabatic bifurcations in nonlinear Hamiltonian systems, Sci. Adv. 5, eaav2372 (2019).

[57] U. Leonhardt, Measuring the Quantum State of Light (Cambridge University Press, Cambridge, 1997).

[58] A. I. Larkin and Y. N. Ovchinnikov, Quasiclassical method in the theory of superconductivity, Sov. Phys. JETP 28, 1200 (1969).

[59] J. Maldacena, S. H. Shenker, and D. Stanford, A bound on chaos, J. High Energ. Phys. 08 (2016) 106.

[60] K. Hashimoto, K. Murata, and R. Yoshii, Out-of-time-order correlators in quantum mechanics, J. High Energ. Phys. 10 (2017) 138.

[61] J. Li, R. Fan, H. Wang, B. Ye, B. Zeng, H. Zhai, X. Peng, and J. Du, Measuring Out-Of-Time-Order Correlators on a Nuclear Magnetic Resonance Quantum Simulator, Phys. Rev. X 7, 031011 (2017).

[62] E. M. Fortes, I. García-Mata, R. A. Jalabert, and D. A. Wisniacki, Gauging classical and quantum integrability through out-of-time-ordered correlators, Phys. Rev. E 100, 042201 (2019).

[63] K. X. Wei, P. Peng, O. Shtanko, I. Marvian, S. Lloyd, C. Ramanathan, and P. Cappellaro, Emergent Prethermalization Signatures in Out-Of-Time Ordered Correlations, Phys. Rev. Lett. 123, 090605 (2019).

[64] T. Akutagawa, K. Hashimoto, T. Sasaki, and R. Watanabe, Out-of-time-order correlator in coupled harmonic oscillators, J. High Energ. Phys. 08 (2020) 013.

[65] K. Hashimoto, K.-B. Huh, K.-Y. Kim, and R. Watanabe, Exponential growth of out-of-time-order correlator without chaos: Inverted harmonic oscillator, J. High Energ. Phys. 11 (2020) 068.

[66] A. Bhattacharyya, W. Chemissany, S. S. Haque, J. Murugan, and B. Yan, The multi-faceted inverted harmonic oscillator: Chaos and complexity, SciPost Phys. Core 4, 002 (2021).

[67] A. Dey, Quantum signatures of chaos in a cavity-QED-based stimulated Raman adiabatic passage, Phys. Rev. A 103, 053704 (2021).

[68] A. W. Harrow, L. Kong, Z.-W. Liu, S. Mehraban, and P. W. Shor, Separation of out-of-time-ordered correlation and entanglement, PRX Quantum 2, 020339 (2021).

[69] X. Mi, P. Roushan, C. Quintana, S. Mandrá, J. Marshall, C. Neill, F. Arute, K. Arya, J. Atalaya, R. Babbush et al., Information scrambling in quantum circuits, Science 374, 1479 (2021).

[70] T. A. Brody, J. Flores, J. B. French, P. A. Mello, A. Pandey, and S. S. M. Wong, Random-matrix physics: Spectrum and strength fluctuations, Rev. Mod. Phys. 53, 385 (1981).

[71] E. Haller, H. Köppel, and L. S. Cederbaum, Uncovering the Transition from Regularity to Irregularity in a Quantum System, Phys. Rev. Lett. 52, 1665 (1984).

[72] M. V. Berry, Quantum chaology, Proc. R. Soc. London A 413, 183 (1987).

[73] M. Berry, Quantum chaology, not quantum chaos, Phys. Scr. 40, 335 (1989).

[74] B. Leimkuhler and S. Reich, Simulating Hamiltonian Dynamics (Cambridge University Press, Cambridge, 2004). 
[75] M. S. Santhanam, V. B. Sheorey, and A. Lakshminarayan, Chaos and exponentially localized eigenstates in smooth Hamiltonian systems, Phys. Rev. E 57, 345 (1998).

[76] M. S. Santhanam and J. N. Bandyopadhyay, Spectral Fluctuations and $1 / f$ Noise in the Order-Chaos Transition Regime, Phys. Rev. Lett. 95, 114101 (2005).

[77] M. S. Santhanam, V. B. Sheorey, and A. Lakshminarayan, Effect of classical bifurcations on the quantum entanglement of two coupled quartic oscillators, Phys. Rev. E 77, 026213 (2008).

[78] E. L. Sibert, III, E. Vergini, R. M. Benito, and F. Borondo, Quantum localization through interference on homoclinic and heteroclinic circuits, New J. Phys. 10, 053016 (2008).

[79] A Hamiltonian system with $n$ degrees of freedom ( $2 n$-dimensional phase space) is integrable if there are $n$ constants of motion [51].

[80] This curve is known as the lemniscate of Bernoulli.

[81] S. B. Lee and M. D. Feit, Signatures of quantum chaos in Wigner and Husimi representations, Phys. Rev. E 47, 4552 (1993).

[82] E. Faleiro, J. M. G. Gómez, R. A. Molina, L. Muñoz, A. Relaño, and J. Retamosa, Theoretical Derivation of $1 / f$ Noise in Quantum Chaos, Phys. Rev. Lett. 93, 244101 (2004).
[83] S. H. Tekur, S. Kumar, and M. S. Santhanam, Exact distribution of spacing ratios for random and localized states in quantum chaotic systems, Phys. Rev. E 97, 062212 (2018).

[84] S. H. Tekur, U. T. Bhosale, and M. S. Santhanam, Higher-order spacing ratios in random matrix theory and complex quantum systems, Phys. Rev. B 98, 104305 (2018).

[85] U. T. Bhosale, S. H. Tekur, and M. S. Santhanam, Scaling in the eigenvalue fluctuations of correlation matrices, Phys. Rev. E 98, 052133 (2018).

[86] S. H. Tekur and M. S. Santhanam, Symmetry deduction from spectral fluctuations in complex quantum systems, Phys. Rev. Research 2, 032063(R) (2020).

[87] R. K. Saini, R. Sehgal, and S. R. Jain, Protection of qubits by nonlinear resonances, arXiv:2011.10329.

[88] S. R. Jain, B. Grémaud, and A. Khare, Quantum modes on chaotic motion: Analytically exact results, Phys. Rev. E 66, 016216 (2002).

[89] S. R. Jain and R. Samajdar, Nodal portraits of quantum billiards: Domains, lines, and statistics, Rev. Mod. Phys. 89, 045005 (2017). 